Rakenteiden Mekaniikka (Journal of Structural Mechanics)

Vol. 52, No 3, 2019, pp. 125-147

http://rakenteidenmekaniikka.journal.fi/index

http://doi.org/10.23998/rm.76025

(C) 2019 The Author

Open access under CC BY 4.0 license

\title{
On continuum damage mechanics
}

\author{
Kari Santaoja ${ }^{1}$
}

Summary. A material containing spherical microvoids with a Hookean matrix response was shown to take the appearance usually applied in continuum damage mechanics. However, the commonly used variable damage $D$ was replaced with the void volume fraction $f$, which has a clear physical meaning, and the elastic strain tensor $\varepsilon^{\mathrm{e}}$ with the damage-elastic strain tensor $\varepsilon^{\text {de }}$. The postulate of strain equivalence with the effective stress concept was reformulated and applied to a case where the response of the matrix obeys Hooke's law. In contrast to many other studies, in the derived relation between the effective stress tensor $\tilde{\boldsymbol{\sigma}}$ and the stress tensor $\boldsymbol{\sigma}$, the tensor $\tilde{\boldsymbol{\sigma}}$ is symmetric. A uniaxial bar model was introduce for clarifying the derived results. Other candidates for damage were demonstrated by studying the effect of carbide coarsening on creep rate.

Key words: spherical voids, Hookean matrix response, effective stress, uniaxial bar model, carbide coarsening, creep

Received 25 October 2018. Accepted 10 July 2019. Published online 31 August 2019.

\section{Introduction}

The difference between the effects of microcrack growth and those of dislocation kinetics on the creep of metals was first recognised by Kachanov [1], who introduced a separate kinematic variable defining locally the microdefect density [2]. Kachanov [1] studied the uniaxial state of stress using a scalar variable to describe damage. Janson and Hult [3] introduced the term "continuous damage mechanics", or CDM. This later became established as "continuum damage mechanics".

${ }^{1}$ Corresponding author: kari.santaoja@aalto.fi 
Today, the theory of continuum damage mechanics is a vital part of many textbooks, as publications [4-10] indicate. Although the list is not comprehensive, it sheds some light on the extensive activity ongoing in the field of damage mechanics. Nonetheless, there is still a need to clarify and reformulate some details within the field, and this forms the scope of the present study.

The current approach to continuum damage mechanics is a classical one describing the influence of microcracks and microvoids on the mechanical properties of materials. In this approach, damage mechanics describes the degradation of material due to microvoids and/or microcracks. The candidates for damage are touched upon at the end of this paper.

Although microcracks and microvoids are discrete objects forming jumps in the material response, their effect is averaged out by continuous functions over a finite volume. Hence the term continuum damage mechanics.

In continuum damage mechanics, the variable damage denoted by $D$ is often introduced. It can be a scalar, vector or tensor of any order. Instead of the variable $D$, it is preferable to introduce variables that are connected to the microstructure of the material, such as the void volume fraction $f$, which is the topic of this paper, and the microcrack densities $Q^{r}$ introduced by Santaoja [11], [12]. Both the void volume fraction $f$ and microcrack densities $Q^{r}$ enter into the theory of continuum thermodynamics as internal variables, being thus a vital part of continuum thermodynamics. By introducing the variable $D$, the writer is often expressing that the source of the degradation of the material is unclear.

The energy expressions including the void volume fraction $f$ or microcrack densities $Q^{r}$ are obtained by using a more or less strict micromechanical survey. It is both preferable and more popular, as several papers [13], [14], [15], [16], [17], [18], [19], [20] indicate, just simply to refer to some of the many studies on this topic.

The Gurson Model [21] for simulation of the response of plastic yield in a porous material is a good example of an old micromechanical model which is still in active use, although now in enhanced form and referred to as Gurson-Tvergaard-Needelman [22].

Since the damage process involves dissipation, continuum thermodynamics is a vital part of validating the model for damage evolution. The key tool for this is the Clausius-Duhem inequality. It is therefore somewhat frustrating that so few damage evolution studies have adopted this approach. There are, however, exceptions [23], [24], [25], [26].

The role of micromechanical modelling and continuum thermodynamics is to set certain restrictions on models. The restrictions help reject models that are inappropriate and avoid introducing a high number of damage variables. A good example of having many damage variables is where the authors had collected 27 tensors from different sources [27]. 


\section{Spherical microvoids with the Hookean matrix response}

Eshelby [28] studied the elastic field in a Hookean matrix material containing an ellipsoidal inclusion. As a special case in his study, the specific damage-elastic Gibbs free energy for a Hookean matrix response with spherical voids $g^{\mathrm{de}}(\boldsymbol{\sigma}, f)$ can be derived and has the following appearance:

$$
g^{\mathrm{de}}(\boldsymbol{\sigma}, f)=\frac{1}{2 \rho_{0}}\left[\frac{1}{3(3 \lambda+2 \mu)}(1+\underline{A} f)[\mathbf{1}: \boldsymbol{\sigma}]^{2}+\frac{1}{2 \mu}(1+\underline{B} f) \mathbf{s}: \mathbf{s}\right] .
$$

In Equation (1) the quantity $\rho_{0}$ is the density of the matrix material in the initial configuration, and $\lambda$ and $\mu$ are the Lamé elastic constants. The notation : stands for the double-dot product operator. Thus, the following holds: $\mathbf{s}: \mathbf{s}=s_{i j} s_{i j}$. The notations $\boldsymbol{\sigma}$ and $\mathbf{s}$ are the stress tensor and the deviatoric stress tensor, respectively. The latter is defined as follows:

$$
\mathbf{s}:=\mathbf{K}: \boldsymbol{\sigma}, \quad \text { where the fourth-order tensor } \quad \mathbf{K}:=\mathbf{I}-\frac{1}{3} \mathbf{1} \mathbf{1} .
$$

The fourth-order identity tensor I and the second-order identity tensor $\mathbf{1}$ are defined to be

$$
\text { Def } \quad \mathbf{I}: \mathbf{c}=\mathbf{c}: \mathbf{I}=\mathbf{c} \quad \text { and } \quad \mathbf{1} \cdot \vec{u}=\vec{u} \cdot \mathbf{1}=\vec{u},
$$

where c and $\vec{u}$ are an arbitrary second-order tensor and an arbitrary vector, respectively. For spherical voids (see Figure 1) the quantities $\underline{A}$ and $\underline{B}$ take the form

$$
\underline{A}=\frac{6 \mu+3 \lambda}{4 \mu} \quad \text { and } \quad \underline{B}=\frac{15(1-v)}{7-5 v}
$$

where $E$ and $v$ are Young's modulus and Poisson's ratio, respectively.

If also the swelling due to the microvoid nucleation and growth and other potential deformation mechanisms are taken into account, the material model for the present study takes the following format:

$$
g(\boldsymbol{\sigma}, f, \ldots, T)=g^{\mathrm{de}}(\boldsymbol{\sigma}, f)+g^{\mathrm{sw}}(\boldsymbol{\sigma}, f)+g^{\mathrm{rest}}(\ldots, T),
$$

where $T$ is the absolute temperature. Expression (1) is derived for a case where the deformation mechanisms modelled in $g^{\text {rest }}(\ldots, T)$ are not present. Thus Partitioning (5) assumes that there is no interaction between the processes described by the models $g^{\mathrm{de}}(\boldsymbol{\sigma}, f)$ and $g^{\text {rest }}(\ldots, T)$. The specific Gibbs free energy associated to porosity swelling $g^{\mathrm{sw}}(\boldsymbol{\sigma}, f)$ is

$$
g^{\mathrm{sw}}(\boldsymbol{\sigma}, f)=\frac{1}{3 \rho_{0}} \mathbf{1}: \boldsymbol{\sigma}\left(f-f_{\mathrm{r}}\right)
$$

where $f_{\mathrm{r}}$ is the reference void volume fraction. The (total) strain tensor $\boldsymbol{\varepsilon}$ and the inelastic strain tensor $\varepsilon^{i}$ are assumed to vanish at the reference state. The same 
holds for the void volume fraction $f$. The terms $g^{\mathrm{de}}(\boldsymbol{\sigma}, f)$ and $g^{\mathrm{sw}}(\boldsymbol{\sigma}, f)$ are not added together, since they model different response mechanisms. The former describes the stiffness reduction due to the microvoids and the latter models swelling due to the microvoid nucleation and growth.

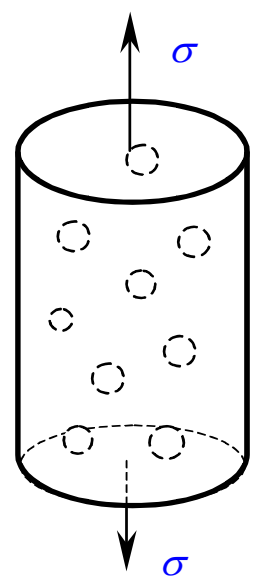

Figure 1. A Hookean matrixresponse with spherical cavities.

For the present set of state variables state equations are

$$
\boldsymbol{\varepsilon}-\boldsymbol{\varepsilon}^{\mathrm{i}}=\rho_{0} \frac{\partial g(\boldsymbol{\sigma}, f, \ldots, T)}{\partial \boldsymbol{\sigma}} \quad \text { and } \quad e=\rho_{0} \frac{\partial g(\boldsymbol{\sigma}, f)}{\partial f} .
$$

In State Equation $(7)_{2}$ the notation $e$ stands for the internal force associated with the void volume fraction $f$.

Substitution of Models (1) and (6) into Partition (5) and further of the result into Expression (7) $)_{1}$ gives

$$
\boldsymbol{\varepsilon}-\boldsymbol{\varepsilon}^{\mathrm{i}}=\rho \frac{\partial g(\boldsymbol{\sigma}, f)}{\partial \boldsymbol{\sigma}}=\frac{1+\underline{A} f}{3(3 \lambda+2 \mu)} \mathbf{1}: \boldsymbol{\sigma} \mathbf{1}+\frac{1+\underline{B} f}{2 \mu} \mathbf{s}+\frac{1}{3}\left(f-f_{\mathrm{r}}\right) \mathbf{1} .
$$

Based on Definitions (2), Constitutive Model (8) can be recast as

$$
\boldsymbol{\varepsilon}-\boldsymbol{\varepsilon}^{\mathrm{i}}=\frac{1+\underline{A} f}{3(3 \lambda+2 \mu)} \mathbf{1} \mathbf{1}: \boldsymbol{\sigma}+\frac{1+\underline{B} f}{2 \mu}\left(\mathbf{I}-\frac{1}{3} \mathbf{1} \mathbf{1}\right): \boldsymbol{\sigma}+\frac{1}{3}\left(f-f_{\mathrm{r}}\right) \mathbf{1} .
$$

Equation (9) can be written as

$$
\boldsymbol{\varepsilon}-\boldsymbol{\varepsilon}^{\mathrm{i}}=\tilde{\mathbf{S}}(f): \boldsymbol{\sigma}+\frac{1}{3}\left(f-f_{\mathrm{r}}\right) \mathbf{1},
$$

where $\tilde{\mathbf{S}}(f)$ is the fourth-order compliance tensor for deformation of the Hookean matrix response with spherical microvoids. It is defined by

$$
\tilde{\mathbf{S}}(f):=\frac{1+\underline{A} f}{3(3 \lambda+2 \mu)} \mathbf{1} \mathbf{1}+\frac{1+\underline{B} f}{2 \mu}\left(\mathbf{I}^{\mathrm{s}}-\frac{1}{3} \mathbf{1} \mathbf{1}\right) .
$$

In Definition (11) the fourth-order identity tensor $\mathbf{I}$ is replaced by the symmetric fourth-order identity tensor $\mathbf{I}^{\mathrm{s}}$. This is possible because the stress tensor $\boldsymbol{\sigma}$ is symmetric. The symmetric fourth-order symmetric identity tensor $\mathbf{I}^{\mathrm{s}}$ is defined as 


$$
\mathbf{I}^{\mathrm{s}}: \mathbf{c}=\mathbf{c}: \mathbf{I}^{\mathrm{s}}=\frac{1}{2}\left(\mathbf{c}+\mathbf{c}^{\mathrm{T}}\right)
$$

where $\mathbf{c}$ is an arbitrary second-order tensor. The tensor transpose of an arbitrary second-order tensor $\mathbf{c}$ is denoted by $\mathbf{c}^{\mathrm{T}}$ and is defined for

$$
\text { Def } \quad \mathbf{c}=c_{i n} \vec{i}_{i} \vec{i}_{n} \text { by } \quad \mathbf{c}^{\mathrm{T}}:=c_{n i} \vec{i}_{i} \vec{i}_{n} \text {. }
$$

The replacement of the tensor $\mathbf{I}$ with the tensor $\mathbf{I}^{\mathbf{s}}$ in Expression (11) makes the fourth-order compliance tensor $\tilde{\mathbf{S}}(f)$ minor (and major) symmetric, which is an important property, since the strain tensors $\boldsymbol{\varepsilon}$ and $\boldsymbol{\varepsilon}^{\mathrm{i}}$ and the stress tensor $\boldsymbol{\sigma}$ are symmetric tensors.

The effective compliance tensor $\tilde{\mathbf{S}}(f)$ can be partitioned into two parts, viz.

$$
\tilde{\mathbf{S}}(f):=\mathbf{S}+\mathbf{S}^{\mathrm{d}}(f),
$$

where the superscript " $d$ " refers to damage. Based on micromechanical evaluation, partition of the effective compliance tensor $\tilde{\mathbf{S}}$ into two parts given in Result (14) is a general result, as the work by Nemat-Nasser and Hori [29, Eq. (4.3.6a)] shows. A similar partition is obtained for rectilinear microcracks in a two-dimensional body [12] and for penny-shaped microcracks in a three-dimensional body [30]. Based on Equations (11) and (14) the compliance tensors $\mathbf{S}$ and $\mathbf{S}^{\mathrm{d}}(f)$ are given by

$$
\mathbf{S}=\frac{1}{3(3 \lambda+2 \mu)} \mathbf{1} \mathbf{1}+\frac{1}{2 \mu}\left(\mathbf{I}^{\mathrm{s}}-\frac{1}{3} \mathbf{1} \mathbf{1}\right)
$$

and

$$
\mathbf{S}^{\mathrm{d}}(f):=\frac{\underline{A} f}{3(3 \lambda+2 \mu)} \mathbf{1} \mathbf{1}+\frac{\underline{B} f}{2 \mu}\left(\mathbf{I}^{\mathrm{s}}-\frac{1}{3} \mathbf{1} \mathbf{1}\right) .
$$

Due to Partition (14) Constitutive Model (10) can be written as

$$
\begin{aligned}
\boldsymbol{\varepsilon}-\boldsymbol{\varepsilon}^{\mathrm{i}}=\tilde{\mathbf{S}}(f): \boldsymbol{\sigma}+\frac{1}{3}\left(f-f_{\mathrm{r}}\right) \mathbf{1} & =\left[\mathbf{S}+\mathbf{S}^{\mathrm{d}}(f)\right]: \boldsymbol{\sigma}+\frac{1}{3}\left(f-f_{\mathrm{r}}\right) \mathbf{1} \\
& =\mathbf{S}: \boldsymbol{\sigma}+\mathbf{S}^{\mathrm{d}}(f): \boldsymbol{\sigma}+\frac{1}{3}\left(f-f_{\mathrm{r}}\right) \mathbf{1} .
\end{aligned}
$$

Based on the second line of Expression (17), the following strain tensors can be introduced:

$$
\boldsymbol{\varepsilon}-\boldsymbol{\varepsilon}^{\mathrm{i}}=\boldsymbol{\varepsilon}^{\mathrm{e}}+\boldsymbol{\varepsilon}^{\mathrm{d}}+\boldsymbol{\varepsilon}^{\mathrm{sw}} \quad \Longrightarrow \quad \boldsymbol{\varepsilon}=\boldsymbol{\varepsilon}^{\mathrm{e}}+\boldsymbol{\varepsilon}^{\mathrm{d}}+\boldsymbol{\varepsilon}^{\mathrm{sw}}+\boldsymbol{\varepsilon}^{\mathrm{i}},
$$

where $\boldsymbol{\varepsilon}^{\mathrm{e}}$ is the elastic strain tensor, $\boldsymbol{\varepsilon}^{\mathrm{d}}$ is the damage strain tensor, $\boldsymbol{\varepsilon}^{\mathrm{sw}}$ is the porosity swelling strain tensor and $\boldsymbol{\varepsilon}^{\mathrm{i}}$ is the inelastic strain tensor. Equations (17) and $(18)_{1}$ give

$$
\boldsymbol{\varepsilon}^{\mathrm{e}}=\mathbf{S}: \boldsymbol{\sigma}, \quad \boldsymbol{\varepsilon}^{\mathrm{d}}=\mathbf{S}^{\mathrm{d}}(f): \boldsymbol{\sigma} \quad \text { and } \quad \boldsymbol{\varepsilon}^{\mathrm{sw}}=\frac{1}{3}\left(f-f_{\mathrm{r}}\right) \mathbf{1} .
$$

It is worth noting that Expression (18) does not include models for all potential deformation mechanisms. The thermal strain tensor $\boldsymbol{\varepsilon}^{\text {Th }}$ could be added to the right 
side of Partition (18). Furthermore, the content of inelastic strain tensor $\boldsymbol{\varepsilon}^{\mathrm{i}}$ has been left open. Expressions (17), (19) ${ }_{1}$ and $(19)_{2}$ allow the introduction of the damageelastic strain tensor $\varepsilon^{\text {de }}$ defined by

$$
\boldsymbol{\varepsilon}^{\mathrm{de}}=\boldsymbol{\varepsilon}^{\mathrm{e}}+\boldsymbol{\varepsilon}^{\mathrm{d}}=\left[\mathbf{S}+\mathbf{S}^{\mathrm{d}}(f)\right]: \boldsymbol{\sigma}=\tilde{\mathbf{S}}(f): \boldsymbol{\sigma} \quad \text { For damaged material. (20) }
$$

Expression $(19)_{3}$ is multiplied by $\mathbf{1}$ : from the left and the following is arrived at:

$$
\mathbf{1}: \boldsymbol{\varepsilon}^{\mathrm{sw}}=\frac{1}{3}\left(f-f_{\mathrm{r}}\right) \mathbf{1}: \mathbf{1}=f-f_{\mathrm{r}} .
$$

Expressions (19)...(21) help to evaluate the physical meaning of the terms in Partition (18). The elastic strain tensor $\boldsymbol{\varepsilon}^{\mathrm{e}}$ gives the response of the spherical-free Hookean deformation. Thus, the elastic strain tensor $\varepsilon^{\mathrm{e}}$ is the deformation of the matrix material, as mentioned in [31] and [32]. The damage strain tensor $\boldsymbol{\varepsilon}^{\mathrm{d}}$ gives the deformation due to the softening of the material caused by the microvoids. When a microcracked material is studied, the damage strain tensor $\boldsymbol{\varepsilon}^{\text {d }}$ gives the deformation due to the softening of the material caused by the microcracks. The role of the damage-elastic strain tensor $\varepsilon^{\text {de }}$ is discussed in the following sections. The porosity swelling strain tensor $\boldsymbol{\varepsilon}^{\text {sw }}$ gives the change of the relative volume of the body with changing porosity $f$, as shown in Equation (21).

\section{Spherical microvoids with the Hookean matrix response when $v=0.2$}

The following can be shown to hold: [cf. Definition (12)]

$$
\mathbf{C}: \mathbf{S}=\mathbf{S}: \mathbf{C}=\mathbf{I}^{\mathrm{S}} \quad \text { and } \quad \mathbf{I}^{\mathrm{s}}: \mathbf{e}=\frac{1}{2}\left(\mathbf{e}+\mathbf{e}^{\mathrm{T}}\right)=\mathbf{e},
$$

where $\mathbf{C}$ is a constitutive tensor for Hookean deformation and $\mathbf{e}$ is an arbitrary symmetric second-order tensor.

Based on Equations (4), the following holds true:

$$
\text { If } \quad v=0.2, \quad \text { then } \underline{A}=\underline{B}=2.0 \text {. }
$$

Substituting Value (23) ${ }_{2}$ into Definition (16) which with Expression (15) gives

$$
\mathbf{S}^{\mathrm{d}}(f)=2 f \mathbf{S} .
$$

Substituting Result (24) into Equation (20) gives

$$
\boldsymbol{\varepsilon}^{\mathrm{de}}=\left[\mathbf{S}+\mathbf{S}^{\mathrm{d}}(f)\right]: \boldsymbol{\sigma} \quad \Longrightarrow \quad \boldsymbol{\varepsilon}^{\mathrm{de}}=(1+2 f) \mathbf{S}: \boldsymbol{\sigma} .
$$

Comparison of Equations (20) and (25) 2 yields

$$
\tilde{\mathbf{S}}(f)=(1+2 f) \mathbf{S} .
$$

Equation $(25)_{2}$ is multiplied by $\mathbf{C}$ : from the left. By taking Equations (22) and the symmetry of the stress tensor $\boldsymbol{\sigma}$ into consideration, the following is arrived at: 


$$
\mathbf{C}: \boldsymbol{\varepsilon}^{\mathrm{de}}=(1+2 f) \mathbf{C}: \mathbf{S}: \boldsymbol{\sigma}=(1+2 f) \mathbf{I}^{\mathrm{s}}: \boldsymbol{\sigma}=(1+2 f) \boldsymbol{\sigma},
$$

which gives

$$
\boldsymbol{\sigma}=(1+2 f)^{-1} \mathbf{C}: \boldsymbol{\varepsilon}^{\mathrm{de}} .
$$

The Maclaurin series of the function $(1+x)^{\alpha}$ reads

$$
(1+x)^{\alpha}=1+\alpha x+\frac{\alpha(\alpha-1)}{2 !} x^{2}+\ldots
$$

Substitution of $\alpha=-1$ and $x=2 f$ into Series (29) which with Equation (28) yields

$$
(1+2 f)^{-1}=1-2 f+(2 f)^{2}+\ldots \quad \Rightarrow \quad \boldsymbol{\sigma} \approx(1-2 f) \mathbf{C}: \boldsymbol{\varepsilon}^{\mathrm{de}} .
$$

The damage-elastic strain $\boldsymbol{\varepsilon}^{\text {de }}$ takes increasing value with an increasing value for the void volume fraction $f$. This means that damage evolution occurs. Thus, if the deformation of the matrix material obeys Hooke's law, according to Equation $(30)_{2}$ the uniaxial stress-strain relation takes the form given by a curve in Figure 2(a).

\section{On strain tensors}

In damage mechanics it is common practice to write the following constitutive equation: \{see e.g. [4], [5] or [33]\}

$$
\sigma=(1-K) E \mathcal{E}^{\mathrm{e}},
$$

where $D$ is the scalar-valued quantity called damage. The relation between the void volume fraction $f$ and the damage $D$ is studied later in this text.

(a)

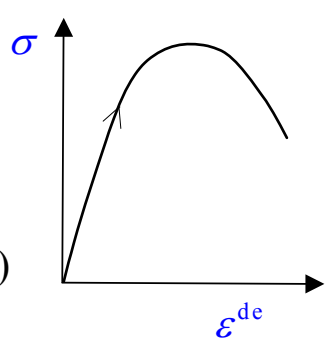

(b)

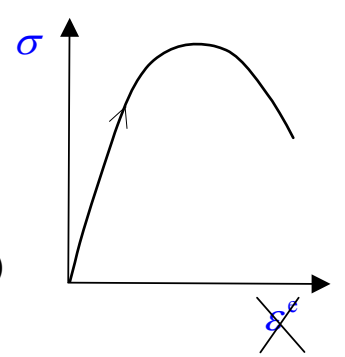

Figure 2. (a) Stress-strain (damage-elastic) curve due to evolution void volume fraction $f$. (b) Stress-strain curve due to damage evolution.

The message of Equation (31) in [5] is given by a drawing similar to that in Figure 2(b). The shape of their stress-strain curve is not the one shown in Figure $2(b)$, but it is not the key concept. The key concept is the difference between the horizontal axes in Figures 2(a) and 2(b).

The shape of the stress-strain curve in Figure 2(b) does not fulfil the 
requirements set for the elastic deformation. According to Malvern [34], a material is called ideally elastic when there is a one-to-one relationship between the state of stress and the state of strain for a given temperature. Equation (19) ${ }_{1}$ gives the stress-strain relation for the elastic (Hookean in this case) deformation and Expression $(30)_{2}$ gives the stress-strain relation for the damage-elastic deformation.

\section{Damage description by the postulate of strain equivalence with the effective stress concept}

The postulate of damage-elastic strain equivalence with the effective stress concept was introduced by Chaboche [32]. Here the definition of the effective stress tensor $\tilde{\boldsymbol{\sigma}}$ by Chaboche is extended for a non-linear material response as follows: If the virgin (undamaged) material obeys the following constitutive equation:

$$
\boldsymbol{\sigma}=f_{1}\left(\boldsymbol{\varepsilon}^{\mathrm{e}}, \text { Virgin }\right) \quad \text { For virgin material, }
$$

then the effective stress tensor $\tilde{\boldsymbol{\sigma}}$ is defined by

$$
\tilde{\boldsymbol{\sigma}}:=f_{1}\left(\boldsymbol{\varepsilon}^{\mathrm{de}}, \text { Virgin }\right) \quad \text { For damaged material. }
$$

It is important to note that Material Models (32) and (33) have an identical functional appearance. Determination of the function $f_{1}$ may be difficult in practice, since damage can occur immediately after loading and no loaded virgin state, within which to determine the function $f_{1}$, exists. Such problems are not studied here.

The terms "Virgin" and "For damaged material" in Definition (33) seem to be contradictory, but this is not the case. "Virgin" indicates that the material properties are taken from an undamaged material, whereas "For damaged material" indicates that the definition is for the quantity, in this case the effective stress tensor $\tilde{\boldsymbol{\sigma}}$ for the damaged material.

Based on the investigation carried out in the section headed "Spherical microvoids with the Hookean matrix response", the following assumption is made:

$$
\boldsymbol{\varepsilon}^{\mathrm{de}}=f_{2}(\boldsymbol{\sigma}, \text { Damaged }) \quad \text { For damaged material. }
$$

Definition (33) is "the effective stress concept". "The postulate of the strain equivalence" tells that the damage-elastic strain tensors $\boldsymbol{\varepsilon}^{\text {de }}$ in Expressions (33) and (34) are equal.

It bears pointing out that the above damage description allows models to be introduced for other deformation mechanisms. In such a case the damage description keeps the form presented above.

A clearer picture of the postulate of strain equivalence with the effective stress concept can be obtained when a particular material model is studied. This is the topic of the next section. 


\section{Analytical relation between the stress tensors $\tilde{\sigma}$ and $\sigma$ for the Hookean matrix response}

Model 1 assumes that the elastic deformation of the matrix material obey's Hooke's law in its virgin state. Thus, Equation (32) yields

$$
\boldsymbol{\sigma}=f_{1}\left(\boldsymbol{\varepsilon}^{\mathrm{e}}, \text { Virgin }\right) \quad \Longrightarrow \quad \boldsymbol{\sigma}=\mathbf{C}: \boldsymbol{\varepsilon}^{\mathrm{e}} \quad \text { For virgin material }
$$

Based on Model 1, the definition for the effective stress $\tilde{\boldsymbol{\sigma}}$ [Definition (33)] reads

$$
\tilde{\boldsymbol{\sigma}}:=f_{1}\left(\boldsymbol{\varepsilon}^{\mathrm{de}}, \text { Virgin }\right) \quad \Longrightarrow \quad \tilde{\boldsymbol{\sigma}}=\mathbf{C}: \boldsymbol{\varepsilon}^{\mathrm{de}} \quad \text { For damaged material. (36) }
$$

When spherical microvoids with the Hookean matrix response were investigated, Expression (20) was derived. Now the result is extended to cover the damaged material in general by dropping the dependency of the effective compliance tensor $\tilde{\mathbf{S}}(f)$ on the void volume fraction $f$. Thus, the following model is written:

Model 2 is assumed to have the following appearance:

$$
\boldsymbol{\varepsilon}^{\mathrm{de}}=f_{2}(\boldsymbol{\sigma}, \text { Damaged }) \quad \Longrightarrow \quad \boldsymbol{\varepsilon}^{\mathrm{de}}=\tilde{\mathbf{S}}: \boldsymbol{\sigma} \quad \text { For damaged material }
$$

Substitution of Equation (37) $)_{2}$ into Definition $(36)_{2}$ leads to

$$
\tilde{\boldsymbol{\sigma}}=\mathbf{C}: \tilde{\mathbf{S}}: \boldsymbol{\sigma} \quad \Longrightarrow \quad \tilde{\boldsymbol{\sigma}}=\mathbf{M}: \boldsymbol{\sigma}, \quad \text { where } \quad \mathbf{M}:=\mathbf{C}: \tilde{\mathbf{S}}
$$

Expressions (38) give an important result for damage mechanics. They show that in the case of non-interacting microvoids and non-interacting microcracks, it is possible to derive an exact analytical relation between the effective stress tensor $\tilde{\boldsymbol{\sigma}}$ and the stress tensor $\boldsymbol{\sigma}$. Thus, no ad hoc model is needed and actually no other model than that given by Expressions (38) can be introduced.

Model $(37)_{2}$ is valid for a Hookean matrix deformation with spherical microvoids [see Equation (20)]. According to Santaoja [11,30,36], it is valid for a Hookean matrix deformation with penny-shaped microcracks and for rectilinear microcracks in a 2D solid [12], although for these latter two cases the damage strain tensor $\boldsymbol{\varepsilon}^{\mathrm{d}}$ has to be made symmetric. Therefore, Equations (38) are valid for these three types of material behaviours. This was the reason for setting Model $(37)_{2}$ as a definition for the damaged material in general. The effective compliance tensors $\tilde{\mathbf{S}}$ for the above three different types of damage are minor and major symmetric. Thus, the effective stress tensor $\tilde{\boldsymbol{\sigma}}$ is symmetric. This is not always the case, as discussed in [37] and [9].

By multiplying Expression $(36)_{2}$ by $\mathbf{S}$ : from the left and by taking Expression $(22)_{1}$ into consideration the following is arrived at:

$$
\mathbf{S}: \tilde{\boldsymbol{\sigma}}:=\mathbf{S}: \mathbf{C}: \boldsymbol{\varepsilon}^{\mathrm{de}} \quad \Longrightarrow \quad \mathbf{S}: \tilde{\boldsymbol{\sigma}}=\mathbf{I}^{\mathrm{s}}: \boldsymbol{\varepsilon}^{\mathrm{de}} \quad \Longrightarrow \quad \boldsymbol{\varepsilon}^{\mathrm{de}}=\mathbf{S}: \tilde{\boldsymbol{\sigma}},
$$

where Definition $(22)_{2}$ and the symmetry of the damage-elastic strain tensor $\boldsymbol{\varepsilon}^{\text {de }}$ are exploited. 
Expression $(37)_{2}$ is multiplied by $\tilde{\mathbf{C}}$ : from left, giving:

$$
\tilde{\mathbf{C}}: \boldsymbol{\varepsilon}^{\mathrm{de}}=\tilde{\mathbf{C}}: \tilde{\mathbf{S}}: \boldsymbol{\sigma} \quad \Longrightarrow \quad \tilde{\mathbf{C}}: \boldsymbol{\varepsilon}^{\mathrm{de}}=\mathbf{I}^{\mathrm{s}}: \boldsymbol{\sigma} \quad \Longrightarrow \quad \boldsymbol{\sigma}=\tilde{\mathbf{C}}: \boldsymbol{\varepsilon}^{\mathrm{de}} .
$$

According to Model 1, i.e. Expression $(36)_{2}$, the relationship between the effective stress $\tilde{\sigma}$ and the damage-elastic strain $\varepsilon^{\text {de }}$ is linear. This is shown in Figure 3(a). It is important to note that the relationship between the effective stress $\tilde{\sigma}$ and the damage-elastic strain $\varepsilon^{\text {de }}$ is also linear in the case of damage evolution.

If there is progressive damage evolution in terms of straining of the material, the stress $\sigma$ vs damage-elastic strain $\varepsilon^{\text {de }}$ may take on a dependence as shown in Figure 2(a) and be copied with ingredients into Figure 3(a). Expression (11) shows that the effective compliance tensor $\tilde{\mathbf{S}}(f)$ takes increasing values with increasing value for the void volume fraction $f$. Thus, Expression $(37)_{2}$ gives the form shown in Figure 2(a) and by a solid line in Figure 3(a). Since growing and nucleating microvoids induce the porosity swelling strain tensor $\boldsymbol{\varepsilon}^{\mathrm{sw}}$, for a porous material the horizontal axis should include the porosity swelling strain $\mathcal{E}^{\mathrm{sw}}$. Therefore, Figure 3(a) is not applicable for microvoids.

(a)

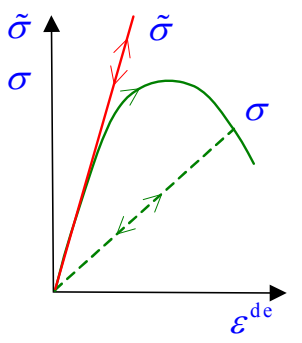

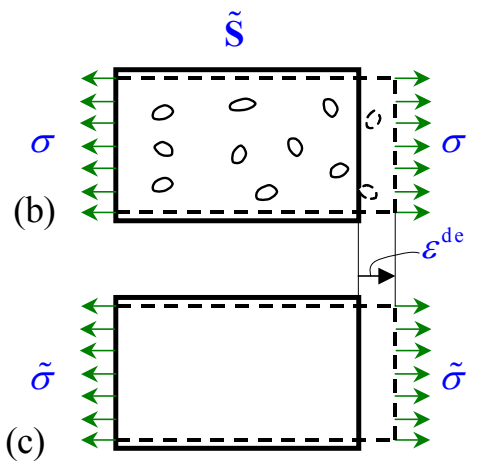

S

Figure 3. (a) Stress-strain curves.

(b) and ( c) Postulate of strain equivalence for a Hookean matrix response.

Now, the damage is assumed to be due to the microcracks. When progressive damage occurs, the strain is not pure elastic strain $\varepsilon^{\mathrm{e}}$. The microcrack formation is a dissipative, i.e. irreversible process. The elastic strain $\varepsilon^{\mathrm{e}}$, on the other hand, describes a reversible process where the unloading path in the stress-strain space takes the same curve, but opposite, to the one during loading. If the microcracking starts after a certain threshold value of deformation, during loading the response of the material is first linear elastic, then when the microcracking occurs, the value for the damage strain $\varepsilon^{\mathrm{d}}$ tends to take increasing values and the curve will deviate from a straight line and finally go downhill, as Figure 3(a) illustrates. So, in the beginning of the unloading there are the elastic strain $\varepsilon^{\mathrm{e}}$ and the damage strain $\varepsilon^{\mathrm{d}}$ within the material. During the unloading procedure the deformation of the matrix material, i.e. the elastic strain $\varepsilon^{\mathrm{e}}$, tends toward zero and the microcracks close, i.e. 
the damage strain $\varepsilon^{\mathrm{d}}$ tends toward zero. Both processes are linear and therefore the unloading path is linear, but it is not pure elastic.

Figures 3(b) and 3(c) sketch the postulate of strain equivalence in a case where the deformation of the virgin material obeys Hooke's law. In Figure 3(b) there is a block of damaged material under tensile stress $\sigma$. Due to the microvoids (or microcracks) the stiffness of the material is reduced and therefore the compliance tensor takes a raised value of $\tilde{\mathbf{S}}$. The stress-strain relation for the damage-elastic response of the material shown in Figure 3(b) follows Equation $(37)_{2}$ and therefore the value of the damage-elastic strain is $\varepsilon^{\text {de }}$. Figure $3(\mathrm{c})$ clarifies the definition of the effective stress tensor $\tilde{\boldsymbol{\sigma}}$ given by Definition (36) ${ }_{2}$ [see Equation (39) ${ }_{3}$ as well]. If the effective stress $\tilde{\sigma}$ acted on the same block of material in its virgin state (i.e. when no microvoids or microcracks existed), the damage-elastic strain $\varepsilon^{\text {de }}$ (the damage strain $\varepsilon^{\mathrm{d}}$ is zero) would take the same value as in the case shown in Figure 3(b).

Next, Poisson's ratio is assumed to be $v=0.2$. Equations (27) and (39) $)_{3}$ are

$$
\mathbf{C}: \boldsymbol{\varepsilon}^{\mathrm{de}}=(1+2 f) \boldsymbol{\sigma} \quad \text { and } \quad \boldsymbol{\varepsilon}^{\mathrm{de}}=\mathbf{S}: \tilde{\boldsymbol{\sigma}} .
$$

Since the effective stress tensor $\tilde{\boldsymbol{\sigma}}$ is symmetric, Equations (41) give

$$
\mathbf{C}: \mathbf{S}: \tilde{\boldsymbol{\sigma}}=(1+2 f) \boldsymbol{\sigma} \quad \Longrightarrow \quad \tilde{\boldsymbol{\sigma}}=(1+2 f) \boldsymbol{\sigma},
$$

where Equations $(22)_{1}, \mathbf{C}: \mathbf{S}=\mathbf{I}^{\mathrm{s}}$, and $(22)_{2}, \mathbf{I}^{\mathrm{s}}: \tilde{\boldsymbol{\sigma}}=\tilde{\boldsymbol{\sigma}}$, are exploited. Series (30), gives

$$
(1+2 f)^{-1} \approx(1-2 f) \quad \Longrightarrow \quad(1+2 f) \approx(1-2 f)^{-1} .
$$

Substitution of Approximation (43) $)_{2}$ into Equation (42) ${ }_{2}$ yields

$$
\tilde{\boldsymbol{\sigma}} \approx \frac{\boldsymbol{\sigma}}{1-2 f} \text {. }
$$

\section{Extended Rabotnov effective stress concept}

When studying creep damage, Rabotnov [38] introduced the concept of the effective stress $\tilde{\sigma}$ using the following uniaxial definition:

$$
\tilde{\sigma}:=\frac{\sigma}{1-D},
$$

where the scalar-valued quantity $D$ is today called damage. For a virgin material $D=0$ and for a fully-damaged material $D=1$.

Comparison of Definition (45) with Analytical Expression (38) provides a tool for deriving the analytical expression for scalar-valued damage $D$ for a Hookean matrix response with spherical microvoids. This is done next.

The concept by Rabotnov, Definition (45), is often extended for a threedimensional state of stress and for isotropic damage as follows: 


$$
\tilde{\boldsymbol{\sigma}}=\frac{\boldsymbol{\sigma}}{1-D} \quad \Longrightarrow \quad \tilde{\boldsymbol{\sigma}}:=\frac{\mathbf{I}: \boldsymbol{\sigma}}{1-D}
$$

In order for Expressions $(46)_{2}$ and $(38)_{1}$ to be equal, the following should hold:

$$
\mathbf{C}: \tilde{\mathbf{S}}=\mathbf{I}(1-D)^{-1} \text {. }
$$

Equation (47) is multiplied by $\mathbf{S}$ : from the left, Property $(22)_{1}$, i.e. $\mathbf{S}: \mathbf{C}=\mathbf{I}^{\mathrm{s}}$, Property (A.1) of Appendix A, i.e. $\mathbf{I}^{\mathrm{s}}: \tilde{\mathbf{S}}=\tilde{\mathbf{S}}$, and Property (A.8) of Appendix A, i.e. $\mathbf{S}: \mathbf{I}=\mathbf{S}$, are exploited. This gives

$$
\mathbf{S}: \mathbf{C}: \tilde{\mathbf{S}}=\mathbf{S}: \mathbf{I}(1-D)^{-1} \quad \Longrightarrow \quad \tilde{\mathbf{S}}=\mathbf{S}(1-D)^{-1} .
$$

According to Equations (23), (24) and (26), only when $v=0.2$ does the following exact result hold true for a Hookean matrix response with non-interacting spherical microvoids:

$$
\mathbf{S}^{\mathrm{d}}(f)=2 f \mathbf{S} \quad \text { and } \quad \tilde{\mathbf{S}}(f)=(1+2 f) \mathbf{S} .
$$

It is important to note that the linear relationship between the compliance tensors $\tilde{\mathbf{S}}$ and $\mathbf{S}$ given in Equation (49) ${ }_{2}$ holds true only when Poisson's ratio takes the value of $v=0.2$. In such a case, Expressions $(48)_{2}$ and $(49)_{2}$ coincide and the extended Rabotnov effective stress concept, Expression (46) $)_{1}$, is valid.

The result derived above means that the extended Rabotnov concept of effective stress, Expressions $(46)_{1}$, is not generally valid for a porous material. This means that at least for a porous material, Expressions (46) $)_{1}$ is an approximation and therefore should be interpreted as a model, as discussed by Santaoja and Kuistiala [39]. This is an important point, since Equation (46) $)_{1}$ is given by many publications, such as in [33], [4] and [5]. Actually, the isotropic damage description requires two damage variables, as pointed out e.g. in [40], [41], [42] and [43].

The relationship between the void volume fraction $f$ and damage $D$ is evaluated. A porous material with a linear elastic matrix response is studied as an example.

Expressions $(48)_{2}$ and $(49)_{2}$ give, for a material with the value $v=0.2$ for Poisson's ratio,

$$
(1-D)^{-1}=(1+2 f) \quad \Longrightarrow \quad D=\frac{2 f}{1+2 f} .
$$

Result $(50)_{2}$ implies that for a low void volume fraction $f$ [Eshelby's theory is based on this assumption], and when the Poisson's ratio $v=0.2$, the following holds:

$$
D \approx 2 f .
$$

The definition for the effective stress $\tilde{\boldsymbol{\sigma}}$, Definition $(36)_{2}$, and the extended Rabotnov effective stress concept, Expression (46), are 


$$
\tilde{\boldsymbol{\sigma}}:=\mathbf{C}: \boldsymbol{\varepsilon}^{\mathrm{de}} \quad \text { and } \quad \tilde{\boldsymbol{\sigma}}=\frac{\boldsymbol{\sigma}}{1-D} .
$$

Equations (52) give

$$
\mathbf{C}: \boldsymbol{\varepsilon}^{\mathrm{de}}=\frac{\boldsymbol{\sigma}}{1-D}, \quad \Longrightarrow \quad \boldsymbol{\sigma}=(1-D) \mathbf{C}: \boldsymbol{\varepsilon}^{\mathrm{de}} .
$$

Result $(53)_{2}$ is widely used in damage mechanics, although the damage-elastic strain tensor $\boldsymbol{\varepsilon}^{\text {de }}$ is usually (if not always) replaced by the elastic strain tensor $\boldsymbol{\varepsilon}^{\mathrm{e}}$. However, Form $(53)_{2}$ is not acceptable, since the variable $D$ does not have any specific physical meaning. Instead, Form $(30)_{2}$, i.e. $\boldsymbol{\sigma} \approx(1-2 f) \mathbf{C}: \boldsymbol{\varepsilon}^{\mathrm{de}}$, should be used, since the void volume fraction $f$ is a well-defined quantity.

\section{Interpretation of the roles of the stress tensors $\tilde{\sigma}$ and $\sigma$ by a uniaxial bar model}

In order to demonstrate the roles of the stress $\sigma$ and the effective stress $\tilde{\sigma}$ the uniaxial model for a damaged material, shown in Figure 4, is evaluated. The model is assumed to be based on $n$ equal tensile bars. The cross-sectional area of a single bar is denoted by $A$. The length of the bars is given by $\ell$. Young's modulus of a single bar is $E$. Variable $m$ gives the number of broken bars. As shown in Figure 4 , the tensile force along an uncracked bar is denoted by $F$ and the force over the entire system is denoted by $N$. The elongation of the bars due to the loading is denoted by $\Delta \ell$. The vertical walls are assumed to form the vertical "boundaries" for the representative volume element RVE. The horizontal boundaries are on the top and bottom of the vertical walls. In order to keep the figure simple, they are not shown in Figure 4.

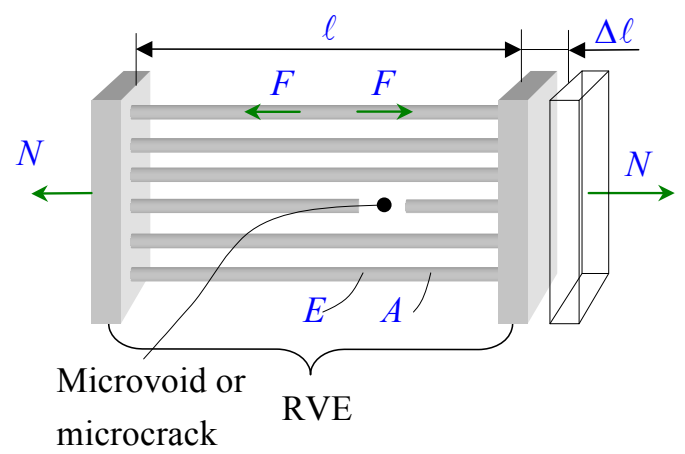

Figure 4. Uniaxial bar model and RVE for evaluation of the stresses $\sigma$ and $\tilde{\sigma}$.

Based on Figure 4, the following is arrived at: 


$$
\sigma^{*}=E \mathcal{E}^{\mathrm{e}}, \quad \text { where } \quad \sigma^{*}=\frac{F}{A} \quad \& \quad \mathcal{E}^{\mathrm{e}}=\frac{\Delta \ell}{\ell} \quad \text { For unbroken bar. }
$$

In Expressions (54) the notation $\sigma^{*}$ stands for the stress in unbroken bars. Since it is evident that all the expressions written for bars are for unbroken bars, the phrase "unbroken" will be left out. Equations $(54)_{1}$ and $(54)_{3}$ give

$$
\sigma^{*}=E \frac{\Delta \ell}{\ell}
$$

Effective stress $\tilde{\sigma}$ is defined to be [cf. Definition $(36)_{2}$, i.e. $\tilde{\boldsymbol{\sigma}}=\mathbf{C}: \boldsymbol{\varepsilon}^{\mathrm{de}}$ ]

$$
\tilde{\sigma}:=E \mathcal{E}^{\mathrm{de}} .
$$

Figure 4 gives

$$
\mathcal{E}^{\mathrm{de}}=\frac{\Delta \ell}{\ell}
$$

As can be seen in Figure 4, Equation (57) is valid for the unbroken bar, since $\mathcal{E}^{\mathrm{de}}=\mathcal{E}^{\mathrm{e}}+\mathcal{E}^{\mathrm{d}}$, where $\mathcal{E}^{\mathrm{d}}=0$, and for the representative volume element RVE.

Substitution of the damage-elastic strain $\mathcal{E}^{\text {de }}$ from Equation (57) into Definition (56) yields

$$
\tilde{\sigma}=E \frac{\Delta \ell}{\ell} .
$$

Comparison of Equations (55) and (58) gives

$$
\sigma^{*}=\tilde{\sigma}
$$

Based on Equation (59), the effective stress $\tilde{\boldsymbol{\sigma}}$ is a microscopic stress in the matrix material between microvoids and/or microcracks. Since the effective stress $\tilde{\boldsymbol{\sigma}}$ takes the same value over all the unbroken bars, the effective stress $\tilde{\boldsymbol{\sigma}}$ is a homogeneous stress field. This means that the effective stress $\tilde{\boldsymbol{\sigma}}$ is an averaged microscopic stress.

\section{Candidates for damage}

The investigation already covered here focused on studying the damage caused by microvoids or microcracks. The obtained expressions focus on cases where increasing deterioration of the elastic stiffness of the material leads to an increasing value for the effective stress tensor $\tilde{\boldsymbol{\sigma}}$. Since the effective stress tensor $\tilde{\boldsymbol{\sigma}}$ is the driving force behind microscopic processes, due to the growing value of the effective stress tensor $\tilde{\boldsymbol{\sigma}}$, some micromechanical processes such as plastic yield, dislocation creep etc. take increasing values. 
There are, however, some other potential sources of damage. The steam pipes of traditional power plants operate at $550^{\circ} \mathrm{C}$ and above. In such conditions, the creep in power plant materials is mainly dislocation creep, and creep resistance is often from carbides that form obstacles to dislocation glide. In order to make the role of the terms in the material model clearer, a constitutive equation is considered, which reads

$$
\varepsilon=\varepsilon^{\mathrm{de}}+\varepsilon^{\mathrm{T}}+\varepsilon^{\mathrm{v}} .
$$

According to Constitutive Equation (60), the (total) strain $\varepsilon$ consists of the damage-elastic strain $\varepsilon^{\text {de }}$, thermal strain $\varepsilon^{\text {Th }}$ and the creep strain $\varepsilon^{\mathrm{v}}$.

The damage to the high-temperature component material has several mechanisms. First, cavities (microvoids) develop on the grain boundaries. As the damage proceeds, the grain boundary cavities coalesce to form microcracks. These damage mechanisms can be modelled by the expressions given in earlier sections of this paper and is included in the damage-elastic strain $\varepsilon^{\text {de }}$. It is not, however, the topic of the current section, which focuses on the creep strain $\varepsilon^{\mathrm{v}}$.

The following material model is written for primary, secondary and tertiary creep:

$$
\dot{\varepsilon}^{\mathrm{v}}=\stackrel{\circ}{\varepsilon}_{\mathrm{re}}\left(\frac{\tilde{\sigma}-\beta}{\sigma_{\mathrm{re}}}\right)^{n} \quad \text { and } \quad \dot{\beta}=c \dot{\varepsilon}^{\mathrm{v}}-b\left(\frac{\omega}{\omega_{\mathrm{re}}}\right)\left(\frac{\beta}{\sigma_{\mathrm{re}}}\right)^{m} e^{\frac{Q}{R}\left(\frac{1}{T_{\mathrm{r}}}-\frac{1}{T}\right)} .
$$

The notations $\stackrel{\circ}{\varepsilon}_{\text {re }}, \sigma_{\text {re }}, n, c, b, \omega_{\text {re }}, m$, and $Q$ are material parameters. The parameter $R$ is the universal gas constant and $T_{\mathrm{r}}$ is the reference temperature. The quantity $\beta$ is the obstacle resistance to dislocation creep and the quantity $\omega$ is the spacing of obstacles (in the slip plane), as shown in Figure 5(a).

The short materials science description for Model (61) is as follows: Since in the present model the deformation due to dislocation glide is described by the viscous strain $\varepsilon^{\mathrm{v}}$, the first term on the right side of Equation $(61)_{2}$, i.e. $c \dot{\varepsilon}^{\mathrm{v}}$, displays the fact that the more dislocations glide, the more they hit the obstacles producing stronger forces onto the carbides. Based on Newton's law of action and reaction, the carbides push back against the dislocations by the same but opposite force. Therefore, the value of the quantity $\beta$ grows with the growing viscous strain $\varepsilon^{\mathrm{v}}$.

The middle part of the second term on the right side of Equation $(61)_{2}$, i.e. $\left(\beta / \sigma_{\mathrm{re}}\right)^{m}$, indicates that the more the dislocations are pushed against carbides, the more they climb over them, with the result that the force on the carbides, $(\sim \beta)$ diminishes with the decreasing number of dislocations pushed onto them. Therefore, the second term on the right side of Equation $(61)_{2}$ has a minus sign.

The spacing of carbides $\omega$ is not a constant, but carbide coarsening is a vital deformation mechanism at elevated temperatures. Since no new material for carbides is available, the spacing of the carbides $\omega$ grows with increasing carbide 
coarsening. Since the resultant force has the form $\tau b^{*} \omega$, the resultant force on the carbides $(\sim \beta)$ grows with carbide coarsening. As the resultant force $\tau b^{*} \omega$ grows, the more dislocations climb over the carbides, which finally leads to a lower value for the force $\beta$. Therefore, the quantity $\omega$ is placed after the minus sign in Equation $(61)_{2}$.

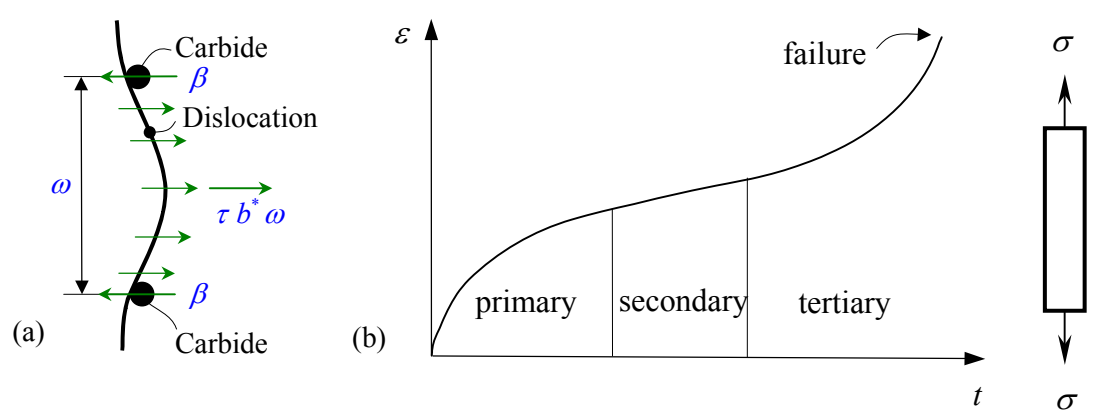

Figure 5. (a) Dislocation hits carbides. (b) Creep curve.

The last exponential term being in Equations (61) is the Arrhenius equation having the form

$$
e^{\frac{Q}{R}\left(\frac{1}{T_{\mathrm{r}}}-\frac{1}{T}\right)}
$$

The equation describes dependence of process rates on temperature. In the present case it describes the vibrations of atoms otherwise known as temperature $T$. 3. The more the atoms vibrate, the higher the temperature $T$. The increasing vibration of the atoms allows the dislocations to climb more easily over the carbides, leading to a higher creep strain rate $\dot{\varepsilon}^{\mathrm{v}}$. At the same time, the carbide coarsening rate $\dot{\omega}$ takes values, since it is dependent on the amount of vibrations of atoms. Thus, the model for the carbide coarsening rate $\dot{\omega}$ should have the form

$$
\dot{\omega} \propto e^{\frac{Q}{R}\left(\frac{1}{T_{\mathrm{r}}}-\frac{1}{T}\right)} .
$$

Increasing operational temperature $T$ can be interpreted to worsen the damage to the components of traditional power plants by these two processes as well.

The above examples demonstrate the variety of potential damage mechanisms. They cannot be modelled simply by introducing a $(1-D)$ term; a detailed investigation of the microscopic processes behind every single mechanism is necessary, and the macroscopic model has to be a description of these microscopic processes.

The nominator $\tilde{\sigma}-\beta$ in Material Model (61) $)_{1}$ expresses the fact that the effective stress $\tilde{\sigma}$ is the driving force behind dislocation glide, i.e. dislocation creep, whereas the force $\beta$ creates an obstacle to it. Actually, the effective shear stress $\tilde{\tau}$ is the driving force, or in the three-dimensional case the deviatoric 
effective stress tensor $\tilde{\mathbf{s}}$ and the von Mises value of the effective stress tensor $J_{\mathrm{vM}}(\tilde{\boldsymbol{\sigma}})$, which should be vital parts of the three-dimensional counterpart to Constitutive Model (61).

Based on the above discussion and Model (61), carbide coarsening increases the creep rate $\dot{\varepsilon}^{\mathrm{v}}$ and it can therefore be interpreted to describe damage to the material. However, the physical processes behind carbide coarsening and their mathematical representations deviate substantially from those that are usually assumed to cause damage to materials.

Based on the above discussion, Model (61) is potentially capable of describing the whole creep curve shown in Figure 5(b).

\section{Discussion and conclusions}

The aim of the present paper was to formulate the theory of continuum damage mechanics for an elastic matrix response with spherical microvoids, penny-shaped microcracks and rectilinear microcracks in a two-dimensional body. The derived approach allows other deformation mechanisms such as porosity swelling and inelastic deformation to be included in the model. Other material mechanisms are briefly discussed as potential candidates for damage.

Based on the work by Eshelby [28], the expression for the specific Gibbs free energy for a Hookean matrix response with spherical microvoids $g^{\text {de }}$ was given. The specific Gibbs free energy for porosity swelling $g^{\text {sw }}$ was introduced as well. Based on these two energy functions a constitutive equation was derived between the damage-elastic strain tensor $\boldsymbol{\varepsilon}^{\mathrm{de}}$ and the stress tensor $\boldsymbol{\sigma}$ in terms of the void volume fraction $f$ and the effective compliance tensor $\tilde{\mathbf{S}}$.

With the introduction to the damage-elastic strain tensor $\boldsymbol{\varepsilon}^{\text {de }}$ the standard form for the widely used material model for damaging materials, i.e.

$$
\sigma=(1-D) E \mathcal{E}^{\mathrm{e}}
$$

was shown to be incapable of describing the damage process. According to the present result, Formula (64) should be replaced by the expression

$$
\boldsymbol{\sigma} \approx(1-2 f) \mathbf{C}: \boldsymbol{\varepsilon}^{\mathrm{de}} .
$$

The criticism is not that the former formula is a scalar equation and the latter expression a tensor-valued equation and that therefore Young's modulus $E$ should be replaced with the constitutive tensor for the Hookean deformation $\mathbf{C}$. The problem with Formula (64) is that the elastic strain (tensor) $\mathcal{E}^{\mathrm{e}}$ has to be replaced with the damage-elastic strain tensor $\boldsymbol{\varepsilon}^{\text {de }}$ and the quantity damage $D$ with the void volume fraction $f$. For microcracked materials the void volume fraction $f$ is not used, but other physically based variables enter into the expressions for damage mechanics. 
A general form was formulated for the description of damage using the postulate of strain equivalence with the effective stress concept. It was written in terms of the damage-elastic strain tensor $\boldsymbol{\varepsilon}^{\mathrm{de}}$, but the formulation allows models to be included for other deformation mechanisms as well. As a special case, the response of a matrix material was assumed to obey Hooke's law. The analytical expression between the effective stress tensor $\tilde{\boldsymbol{\sigma}}$ and the stress tensor $\boldsymbol{\sigma}$ was derived for a case where one of the deformation mechanisms of the matrix material obeys Hooke's law. The expression was shown to take the form

$$
\tilde{\boldsymbol{\sigma}}=\mathbf{C}: \tilde{\mathbf{S}}: \boldsymbol{\sigma} \quad \Longrightarrow \quad \tilde{\boldsymbol{\sigma}}=\mathbf{M}: \boldsymbol{\sigma}, \text { where } \quad \mathbf{M}:=\mathbf{C}: \tilde{\mathbf{S}}
$$

Since the effective compliance tensor $\tilde{\mathbf{S}}$ is a fourth-order minor (and major) symmetric tensor, the effective stress tensor $\tilde{\boldsymbol{\sigma}}$ is a symmetric tensor, which is not always the case in damage mechanics, as expressed in [37] and [9].

The roles of the stress tensors $\tilde{\boldsymbol{\sigma}}$ and $\boldsymbol{\sigma}$ were examined with a uniaxial bar model. Based on the model, the effective stress tensor $\tilde{\boldsymbol{\sigma}}$ was interpreted to be an averaged microscopic stress and it is defined in the matrix material between microvoids and/or microcracks. The stress tensor, on the other hand, is a macroscopic averaged stress. Thus, the effective stress tensor $\tilde{\boldsymbol{\sigma}}$ is the driving force behind the micromechanical processes such as creep and plastic yield.

If brittle cracking, for example, of the matrix material is modelled, the elastic strain tensor $\boldsymbol{\varepsilon}^{\mathrm{e}}$ has to be a vital part of the model, since it gives the deformation of the matrix material.

Finally, the role of carbide coarsening was evaluated as a candidate for damage of materials. A detailed evaluation of the terms in the creep equation was carried out.

\section{References}

[1] L. M. Kachanov. O vremeni razrusheniya v usloviyah polzuchesti. [Time of the rupture process under creep conditions.]. Izw. Akad. Nauk. SSSR, Otd. Teh. Nauk, (8): 26-31, 1958. (In Russian).

[2] D. Krajcinovic. Continuum Damage Mechanics. Applied Mechanics Reviews, 37(1): 1-6, 1984.

[3] J. Janson and J. Hult. Fracture mechanics and damage mechanics: a combined approach. Journal de Mécanique appliquée, 1(1) : 69-84, 1977.

[4] J. Lemaitre. A Course on Damage Mechanics. Springer-Verlag, Berlin, 1992.

[5] J. Skrzypek and A. Ganczarski. Modelling of Material Damage and Failure of Structures. Springer-Verlag, Berlin, 1999.

[6] D. Krajcinovic. Damage Mechanics. Second impression. Elsevier, Amsterdam. 2003. 
[7] J. J. Skrzypek and A. W. Ganczarski. (eds.). Anisotropic Behaviour of Damaged Materials. Springer-Verlag, Berlin. 2003.

[8] G. Z. Voyiadjis and P. I. Kattan. Damage Mechanics. CRC Press, 6000 Broken Sound Parkway NW, Suite 300, Boca Raton, 2005.

[9] S. Murakami. Continuum Damage Mechanics: A Continuum Mechanics Approach to the Analysis of Damage and Fracture. Springer, Dordrecht, 2012. doi: 10.1007/978-94-007-2666-6

[10] L. Dormieux and K. Djimedo. Micromechanics of Fracture and Damage. John Wiley \& Sons, Inc., New York, 2016.

[11] K. Santaoja. Thermodynamic formulation of a material model for microcracking applied to creep damage. Proceedia Materials Science, 3:1179-1184, 2014. doi:10.1016/j.mspro.2014.06.192

[12] K. Santaoja and J. N. Reddy. Material Model for Creep-Assisted Microcracking Applied to S2 Sea Ice. Journal of Applied Mechanics, 83(11): 111002, 2016. doi: 10.1115/1.4034345

[13] J. Kullaa. Micromechanics of multiple cracking: Part I. Fibre analysis. Journal of Materials Science, 33(16): 4213-4234, 1998.

[14] J. Kullaa. Micromechanics of multiple cracking: Part II. Statistical tensile behaviour. Journal of Materials Science, 33(16): 4213-4234, 1998.

[15] Z. Yan, Q. Chen, H. Zhu, J.W. Ju, S. Zhou and Z. Jiang. A multi-phase micromechanical model for unsaturated concrete repaired using the electrochemical deposition method. International Journal for Solids and Structures, 50: 3875-3885, 2013. doi: 10.1016/j.ijsolstr.2013.07.020

[16] L. Longbiao. Micromechanical Modelling for Tensile Behaviour of Carbon Fiber - Reinforced Ceramic - Matrix Composites. Applied Composite Materials, 22: 773-790, 2015. doi: 10.1007/s10443-014-9435$\mathrm{y}$

[17] C. Goidescu, H. Welemane, O. Pantalé, M. Karama and D. Kondo. Anisotropic unilateral damage with initial orthotropy: A micromechanicsbased approach. International Journal of Damage Mechanics, 24(3): 313337, 2015. doi: 10.1177/1056789514532299

[18] Y. Zhang, Z.-g. Yan, J.W. Ju, H.-h. Zhu and Q. Chen. A multi-level micromechanical model for elastic properties of hybrid fiber reinforced concrete. Construction and building Materials, 152: 804-817, 2017. doi: 10.1016/j.conbuildmat.2017.07.024

[19] W. Jin and C. Arson. Micromechanics based discrete damage model with multiple non-smooth yield surfaces: Theoretical formulation, numerical implementation and engineering applications. International Journal of Damage Mechanics, 27(5): 611-639, 2018. doi: $10.1177 / 1056789517695872$

[20] Z.-g Yan, Y. Zhang, J.W. Ju, Q. Chen and H.-h. Zhu. An equivalent elastoplastic damage model based on micromechanics for hybrid fiber- 
reoinforced composites under uniaxial tension. International Journal of Damage Mechanics, 28(1): 79-117, 2019. doi: $10.1177 / 1056789517744425$

[21] A.L. Gurson. Continuum theory of ductile rupture by void nucleation and growth: Part 1 - Yield criteria and flow rules for porous ductile media. Journal of Engineering Materials and Technology, 99(1): 2-15, 1977.

[22] M. Djouabi, A. Ati and P.-Y. Manach. Identification strategy influence of elastoplastic behavior law parameters on Gurson-Tvergaard-Needleman damage parameters: Application to DP980 steel. International Journal of Damage Mechanics, 28(3): 427-454, 2019.

[23] R.K. Abu and M.K. Darabi. A thermodynamic framework for constitutive modelling of time- and rate-dependent materials, Part I: Theory. International Journal of Plasticity, 34: 61-92, 2012. doi: 10.1016/j.ijplas.2012.01.002

[24] H. Zhu and L. Sun. A viscoelastic-viscoplastic damage constitutive model for asphalt mixtures based on thermodynamics. International Journal of Plasticity, 40: 81-100, 2013. doi: 10.1016/j.ijplas.2012.07.005

[25] J.-Y. Wu and M. Cervera. A thermodynamically consistent plastic-damage framework for localized failure in quasi-brittle solids: Material model and strain localization analysis. International Journal for Solids and Structures, 88-89: 227-247, 2016. doi: 10.1016/j.ijsolstr.2016.03.005

[26] H. Shahsavari, R. Naghdabadi, M. Baghani and S. Sohrabpour. A viscoelastic-viscoplastic constitutive model considering damage evolution for time dependent materials: Application to asphalt mixes. International Journal of Damage Mechanics, 25(7): 921-942. doi: 10.1177/105678951665806

[27] G.V. Voyiadis, M.A. Yousef and P.I. Kattan. New Tensors for Anisotropic Damage in Continuum Damage Mechanics. Journal of Engineering Materials and Science, 134: 021015-1 - 021015-7. doi: $10.1115 / 1.4006067$

[28] J. D. Eshelby. The determination of the elastic field of an ellipsoidal inclusion, and related problems. Proceedings of the Royal Society of London, A241: 376-396, 1957.

[29] S. Nemat-Nasser and M. Hori. Micromechanics: Overall properties of heterogeneous materials. Elsevier, Amsterdam, 1993.

[30] K. Santaoja. Continuum damage mechanics approach to describe multidirectional microcracking of ice. In: Sinha, N.K., Sodhi, D.S. \& Chung, J.S. (Eds.), Proceedings of the Eight International Conference on Offshore Mechanics and Arctic Engineering. The Hague, The Netherlands: Vol. IV 55-65, 1989.

[31] Z. Ren, L. Wang and X. Peng. Micromechanics model of gas saturated coal weakened by elliptical microcracks. International Journal of Damage 
Mechanics, 26(7): 1043-1060, 2017. doi: 10.1177/1056789516648368

[32] W. Jin and C. Arson. Micromechanics based discrete damage model with multiple non-smooth yield surfaces: Theoretical formulation, numerical implementation and engineering applications. International Journal of Damage Mechanics, 27(5): 611-639. doi: 10.1177/1056789517695872

[33] J. Lemaitre and J.-L. Chaboche. Mechanics of solid materials. Cambridge University Press, New York, 1990.

[34] L. E. Malvern. Introduction to the Mechanics of a Continuous Medium. Prentice-Hall, Englewood Cliffs, New Jersey, 1969.

[35] J.-L. Chaboche. Description thermodynamique et phénoménologique de la viscoplasticité cyclique avec endommagement. Chatillon, France: Office National d'Etudes et de Recherches Aérospatiales. Publication no. 1978-3, Chatillon. (In French)

[36] K. Santaoja. Mathematical modelling of deformation mechanisms in ice. Espoo, Finland: Technical Research Centre of Finland, Research Reports no. 676, Espoo.

[37] J. Betten. Net-Stress Analysis in Creep Mechanics. Ingenieur-Archiv, (52): 405-419, 1982.

[38] Y. N. Rabotnov. Creep rupture. In: Hetényi. M. \& Vincent W. G. (Eds.). Proceedings of the XII International Congress of Applied Mechanics. Stanford, USA: 342-349, 1968. Springer-Verlag, Berlin, 1969.

[39] K. Santaoja and A. Kuistiala. Material models for Hookean Materials with voids or cracks. In: W. Gutkowski \& T. A. Kowalewski (Eds.), Proceedings of the 21st International Congress of Theoretical and Applied Mechanics. Warsaw, Poland: 237, 2004.

[40] Q.S. Zheng and J. Betten. On damage effective stress and equivalence hypothesis. International Journal of Damage Mechanics, 5(3): 219-240, 1996. doi: 10.1177/105678959600500301

[41] A. Cauvin and R.B. Testa. Damage mechanics: basic variables in continuum theories. International Journal of Solids and Structures, 36: 747-761, 1999.

[42] C.L. Chow and Y. Wei. Constitutive Modeling of Material Damage for Fatigue Failure Prediction. International Journal of Damage Mechanics, 8: 355-375, 1999.

[43] Q. Yang and K. Leng. A microplane-based anisotropic damage effective stress. International Journal of Damage Mechanics, 23(2): 178-191, 2014. doi: $10.1177 / 10567895134888009$ 


\section{Appendix A}

Theorem 1: The following holds:

$$
\mathbf{I}^{\mathrm{s}}: \tilde{\mathbf{S}}=\tilde{\mathbf{S}}
$$

Proof: The fourth-order symmetric identity tensor $\mathbf{I}^{\mathrm{s}}$ is defined as

Def

$$
\mathbf{I}^{\mathrm{s}}: \mathbf{c}=\mathbf{c}: \mathbf{I}^{\mathrm{s}}=\frac{1}{2}\left(\mathbf{c}+\mathbf{c}^{\mathrm{T}}\right)
$$

where $\mathbf{c}$ is an arbitrary second-order tensor. The tensor transpose of an arbitrary second-order tensor $\mathbf{c}$ is denoted by $\mathbf{c}^{\mathrm{T}}$ and it is defined for

$$
\text { Def } \quad \mathbf{c}=c_{i n} \vec{i}_{i} \vec{i}_{n} \quad \text { by } \quad \mathbf{c}^{\mathrm{T}}:=c_{n i} \vec{i}_{i} \vec{i}_{n} \text {. }
$$

Based on Definition (A.2) the component form of the tensor $\mathbf{I}^{\mathrm{s}}$ reads

$$
\mathbf{I}^{\mathrm{s}}=\frac{1}{2}\left(\delta_{i k} \delta_{j l}+\delta_{i l} \delta_{j k}\right) \vec{i}_{i} \vec{i}_{j} \vec{i}_{k} \vec{i}_{l}
$$

Kronecker delta $\delta_{i j}$ is defined to be zero when $i$ and $j$ differ, and to be unity when $i=j$, viz.

$$
\delta_{i j}:=\left\{\begin{array}{l}
0 \text { when the values for } i \text { and } j \text { differ. E.g. } \delta_{10}=0 \\
1 \text { when } i \text { and } j \text { take equal values. E.g. } \delta_{11}=1 .
\end{array}\right.
$$

Based on Form (A.4) the following can be written:

$$
\begin{aligned}
\mathbf{I}^{\mathrm{s}}: \mathbf{A} & =\frac{1}{2}\left(\delta_{i k} \delta_{j l}+\delta_{i l} \delta_{j k}\right) \vec{i}_{i} \vec{i}_{j} \vec{i}_{k} \vec{i}_{l}: A_{s t u w} \vec{i}_{s} \vec{i}_{t} \vec{i}_{u} \vec{i}_{w} \\
& =\frac{1}{2}\left(\delta_{i k} \delta_{j l}+\delta_{i l} \delta_{j k}\right) \vec{i}_{i} \vec{i}_{j} A_{s t u w} \delta_{k s} \delta_{l t} \vec{i}_{u} \vec{i}_{w} \\
& =\frac{1}{2}\left(\delta_{i k} \delta_{j l}+\delta_{i l} \delta_{j k}\right) \vec{i}_{i} \vec{i}_{j} A_{k l u w} \vec{i}_{u} \vec{i}_{w}=\frac{1}{2}\left(A_{i j u w}+A_{j i u w}\right) \vec{i}_{i} \vec{i}_{j} \vec{i}_{u} \vec{i}_{w},
\end{aligned}
$$

where $\mathbf{A}$ is an arbitrary fourth-order tensor. Since the tensor $\tilde{\mathbf{S}}$ is a minor symmetric tensor, see Expressions (14)...(16), Manipulation (A.6) gives the following result:

$$
\mathbf{I}^{\mathrm{s}}: \tilde{\mathbf{S}}=\tilde{\mathbf{S}} \text {. }
$$

Result (A.7) proves Theorem (A.1).

Theorem 2: The following holds:

$$
\mathbf{S}: \mathbf{I}=\mathbf{S} .
$$

Proof: The fourth-order identity tensor $\mathbf{I}$ is defined as

$$
\text { Def } \mathbf{I}: \mathbf{c}=\mathbf{c}: \mathbf{I}=\mathbf{c},
$$

where $\mathbf{c}$ is an arbitrary second-order tensor. The fourth-order identity tensor $\mathbf{I}$ is 
given in terms of its components as follows:

$$
\mathbf{I}=\delta_{i k} \delta_{j l} \vec{i}_{i} \vec{i}_{j} \vec{i}_{k} \vec{i}_{l} \quad \text { or } \quad \mathbf{I}=I_{i j k l} \vec{i}_{i} \vec{i}_{j} \vec{i}_{k} \vec{i}_{l} \text {, where } I_{i j k l}=\delta_{i k} \delta_{j l} \text {. }
$$

Based on Expression (A.10) ${ }_{1}$, the following is arrived at:

$$
\begin{aligned}
\mathbf{S}: \mathbf{I} & =S_{s t u w} \vec{i}_{s} \vec{i}_{t} \vec{i}_{i} \vec{i}_{w}: \delta_{i k} \delta_{j l} \vec{i}_{i} \vec{i}_{j} \vec{i}_{k} \vec{i}_{l}=S_{s t u w} \delta_{u i} \delta_{w j} \delta_{i k} \delta_{j l} \vec{i}_{s} \vec{i}_{t} \vec{i}_{k} \vec{i}_{l} \\
& =S_{s t u w} \delta_{u k} \delta_{w l} \vec{i}_{s} \vec{i}_{t} \vec{i}_{k} \vec{i}_{l}=S_{s t k l} \overrightarrow{\vec{i}}_{s} \vec{i}_{t} \vec{i}_{k} \vec{i}_{l}=\mathbf{S} .
\end{aligned}
$$

Result (A.11) proves Theorem (A.8)

\author{
Kari Santaoja \\ Aalto University \\ Department of Mechanical Engineering \\ P.O.Box 14300, 00760 Aalto \\ kari.santaoja@aalto.fi
}

